\title{
Numerical analysis of varies geometries on dynamic analysis on the gravity platform
}

Análisis numérico de diferentes geometrías en el análisis dinámico en la plataforma de gravedad.

Author:

Tina Rezaei ${ }^{1, *}$

Atefeh Mosahebi Mohammadi ${ }^{2}$

Elham Mina ${ }^{3}$

Mohammad Reza Kavianpour ${ }^{4}$

SCIENTIFIC RESEARCH

How to cite this paper:

Rezaei. T., Mohammadi. A.M., Mina. E., Kavianpour. M.R. Numerical analysis of varies geometries on dynamic analysis on the gravity platform. Tehran, Iran. Innovaciencia. 2019; 7 (2): 1-12. DOI:

http://dx.doi.org/10.15649/2346075X.764

Reception date:

Received: 15 February 2019

Accepted: 2 May 2019

Published: 25 October 2019

Keywords:

gravity-based structures (GBS) hydrodynamic, Ansys Aqwa, JONSWAP, rocking fluctuation

\begin{abstract}
On Gravity platform Dynamic Response is a support structure held in place by gravity. Given that the gravity platforms are supported by the sea bed but not attached to the ground, the motion of them is known as a rocking fluctuation. During the fluctuations, the platform maybe overturned if the angle is large. In this study, using Ansys Aqwa hydrodynamic software and analytical methods with regard to the performance, the dynamic interaction of platform's geometry on hydrodynamic forces have been simulated. The objective of this study was to analyze the hydrodynamic parameters of the sea and rocking fluctuations of gravity platforms under the impact of regular wave's moment considering the soil mechanics and hydrodynamic features of the structure. In order to achieve the objective the hydrodynamic forces using numerical simulations and analytical methods for one column and three hollow columns platforms were analyzed. Finally, responses of the platform to irregular waves were studied using numerical simulation. The results showed that with the increasing of the depth, the impact of wave's force and moment on the base of platform are reduced through exponential relationship. The reductions are due to the effective depth that is equal to half the wave length. The results suggest that the response of the rocking motion of gravity platform shows significant changes in relation to height and wavelength. Based on the curves fitted to the data of the fluctuation angle, sustainability of the platform in the rocking motion can be thoroughly and completely investigated.
\end{abstract}

Corresponding author: Tina Rezaei Email: tina.r_2010@yahoo.com

1 M.Sc. Student of Hydraulic Structures, Azad University, Science and Research Branch, Faculty of Water Engineering, Tehran, Iran. Email: tina.r_2010@yahoo.com

2 M.Sc. Student of Hydraulic Structures, Azad University, Science and Research Branch, Faculty of Water Engineering, Tehran, Iran

3 Ph.D Student of Hydraulic Structures, Tarbiat Modares University, Faculty of Environmental Civil Engineering, Tehran, Iran

4 Professor, K.N.T. University of Technology, Faculty of Civil Engineering, Tehran, Iran 


\section{INTRODUCTION}

The gravity base structure is an offshore concrete structure was based on a cellular base with circular cells and one to four hollow columns (shafts), and thus had the advantage of a slim shape through the wave zone.

Gravity-based structures (GBS) are often constructed in fjords since their protected area and sufficient depth are very desirable for construction. Prior to deployment, a study of the seabed will have been done in order to ensure it can withstand the vertical load exerted on it by that structure. The gravity platforms are supported by the sea bed but not attached to the ground, the motion of them is known as a rocking fluctuation. During the fluctuations, the platform maybe overturned if the angle is large. In this study, using hydrodynamic software and analytical methods with regard to the performance, the dynamic interaction of platform's geometry on hydrodynamic forces have been simulated. The objective of this study was to analyze the hydrodynamic parameters of the sea and rocking fluctuations of gravity platforms under the impact of regular wave's moment considering the soil mechanics and hydrodynamic features of the structure. In order to achieve the objective the hydrodynamic forces using numerical simulations and analytical methods ${ }^{[7]}$ of Mac Cami ${ }^{[3]}$ foch and Chakrabarty ${ }^{[6]}$ for one column and Three hollow columns platforms were analyzed.

\section{MATERIALS AND METHODS}

In this study, using Ansys Aqwa software, the equations of fluid flow including the equations of continuity and momentum were simulated regarding the non-compressibility by boundary element method. The effects of the sea bed on the depreciation of the rocking fluctuation of the construct and hydrodynamic properties were considered for a gravity construct with wavelengths larger than the diameter of the structure using scattering theory around the structure. The study is based on the following assumptions and if other conditions are considered, mathematical relationships need to be corrected. Linear wave theory, harmonic regular waves, rigid gravity platform of one column and Three hollow columns. bases are regarded. The gravity platform is not attached to the soil ${ }^{[1]}$ and wave-induced force is obtained using Erie linear wave theory $[2],[4,[5]$ for a regular wave. Since the geographical location of platforms is located in the North Sea according Figure (1), the environmental conditions and specifications of Concept definition and project organization are as follows:

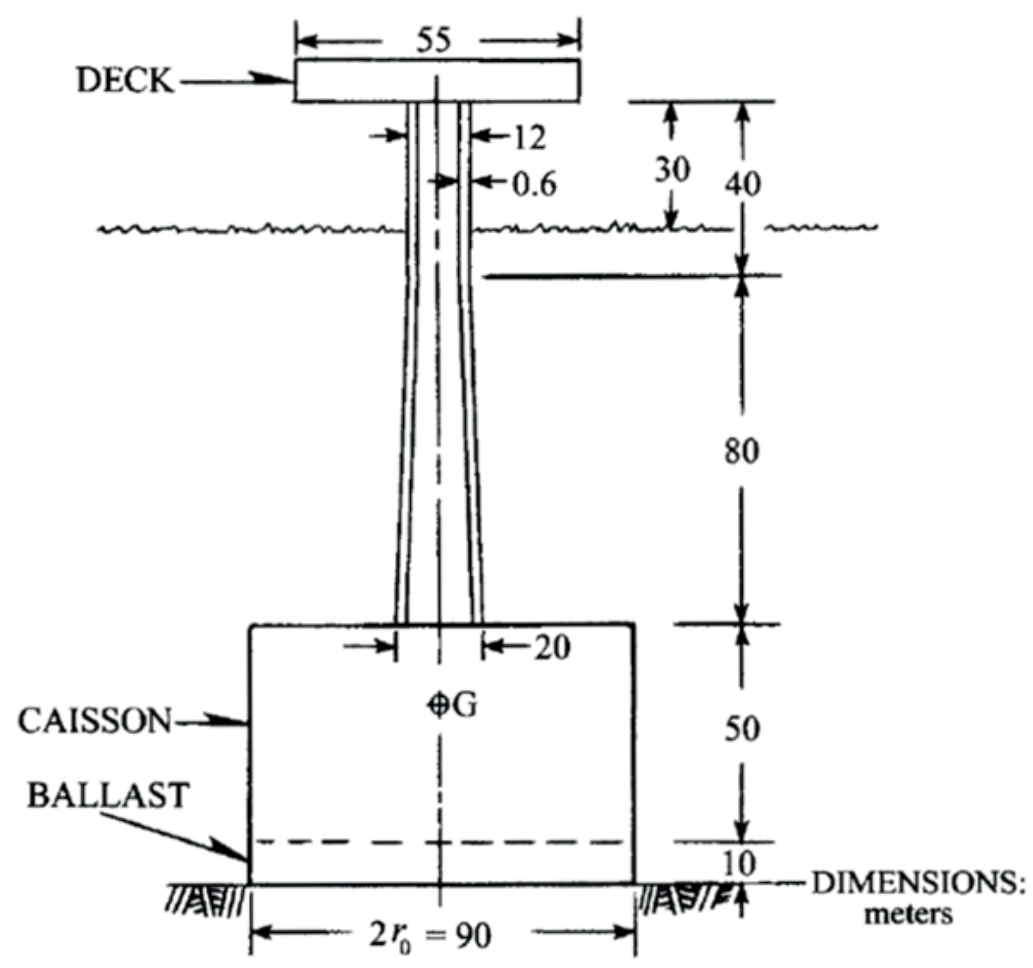

Figure 1. A view of the one column platform 
Geometry \& Environmental modeling Property:

$\rho=1025^{\mathrm{kg}} / \mathrm{m}^{3}, g=9.81 \mathrm{~m} / \mathrm{s}^{2}, H_{0}=6 \mathrm{~m}$,

$d=150 m, T=8 s, \lambda=100 m)$

$\mathrm{k}=\frac{2 \pi}{\lambda}=0.062 \mathrm{~m}^{-1}, \omega=\frac{2 \pi}{T}=0.7853\left(\frac{\mathrm{rad}}{\mathrm{s}}\right)$

\subsection{The governing equations}

The design forces acting on offshore platforms are caused by the wind, sea currents, and waves, which exert great forces on the submerged parts of offshore platforms. Due to the waved-induced velocity and acceleration of fluid particles, forces act on the object body. When the object size is larger than the wave length, the wave shape completely changes after colliding with the object. In this case, Laplace's equation, which was obtained from the wave hydrodynamics discussion, should be used as the governing equation along with the condition of lack of entrance of a flow into the object. This new condition creates diffracted waves, which also exert forces on the object similar to the main waves. The total forces acting on the object will be the resultant of these forces and forces exerted on the object by the main waves. To analyze the diffraction phenomenon and calculate the force acting on the platform abutments (with different geometries), analytical methods were employed and a summary of McKemi's analytical solution for calculation of forces acting on a circular abutment as well as Chakrabarti's analytical solution for calculation of forces acting on several circular abutments was provided ${ }^{[8]}$. In general, to investigate the total field's potential scattering $\Phi_{\mathrm{t}}$ is considered as the total potential of incident waves $\Phi_{i}$ and the potential of Scatter wave $\Phi_{\text {s. }}$.

$\Phi_{t}=\Phi_{i}+\Phi_{s}$

\subsubsection{Mc Kemi's analytical solution}

For a Condeep gravity base structure (one column platform) simplified calculations can be performed for McKemi's analytical solution and numerical model as Figure 2, according this:

$$
\begin{aligned}
& \Phi_{i}=A \frac{\cosh (k(d+z))}{\cosh (k d)}\left[\sum_{m=0}^{\infty} \beta_{m} J_{m}(k r) \cos (m \theta)\right] \cos (\omega-\delta) \\
& \Phi_{s}=A \frac{\cosh k(d+z)}{\cosh (k d)}\left[\sum_{m=0}^{\infty} \beta_{m} B_{m} H^{1}{ }_{m}(k r) \cos (m \theta)\right] \cos (\omega t-\delta) \\
& B_{m}=-\frac{J_{m}^{\prime}(k a)}{H^{1}{ }_{m}(k a)} \\
& \Phi_{t}=A \frac{\cosh k(d+z)}{\cosh (k d)}\left[\sum_{m=0}^{\infty} \beta_{m}\left[J^{\prime}{ }_{m}(k r)-\frac{J_{m}^{\prime}(k a)}{H^{1}{ }_{m}(k a)} H_{m}(k r)\right] \cos (m \theta)\right] \cos (\omega t-\delta)
\end{aligned}
$$




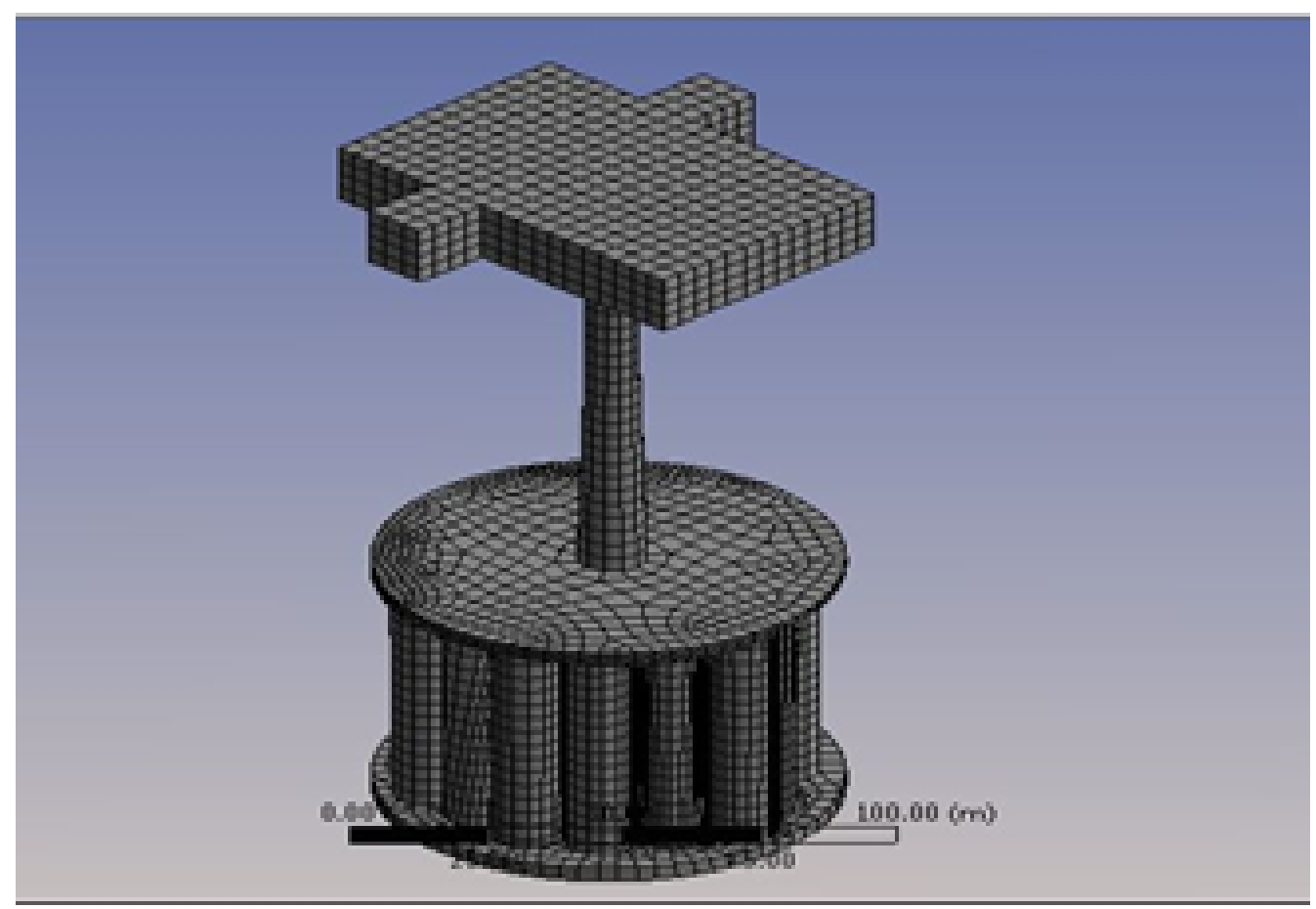

Figure 2. Modeling in AQWA Gravity Base Structure GBS for one column platform

\subsubsection{Chakrabarti's analytical solution}

For a Con deep gravity base structure (Three hollow performed for Chakrabarti's analytical solution and columns platform) simplified calculations can be numerical model as Figure 3, according this:

$$
\begin{gathered}
\Phi_{\mathrm{i}}^{\delta}=\frac{\mathrm{igH}}{2 \omega} \frac{\cosh (\mathrm{k}(\mathrm{d}+\mathrm{z}))}{\cosh (\mathrm{kd})} \mathrm{e}^{\mathrm{ikr} \mathrm{r}_{0} \cos \left(\theta_{\mathrm{i} \delta}-\theta_{\mathrm{w}}\right)} \\
\sum_{n=-\infty}^{\infty} J_{n}\left(k r_{\delta}\right) e^{i\left[n\left(\theta_{\delta}-\theta_{w}+\frac{\pi}{2}\right)-\omega t\right]} \\
\Phi_{\mathrm{i}}^{\delta}=\frac{\mathrm{igH} \cosh (\mathrm{k}(\mathrm{d}+\mathrm{z}))}{\cosh (\mathrm{kd})} \sum_{\mathrm{n}=-\infty}^{\infty} \mathrm{A}_{\mathrm{n}}^{\delta} \mathrm{H}_{\mathrm{n}}^{1}\left(\mathrm{kr}_{\delta}\right) \mathrm{e}^{\mathrm{i}\left[\mathrm{n} \theta_{\delta}-\omega \mathrm{t}\right]} \\
\Phi_{t}^{\delta}=\frac{i g H}{2 \omega} \frac{\cosh (k(d+z))}{\cosh (k d)}\left\{\sum_{n=-\infty}^{\infty} J_{n}\left(k r_{\delta}\right) e^{i k r_{0 \delta} \cos \left(\theta_{0 \delta}-\theta_{w}\right)} e^{i n\left(\theta_{\delta}-\theta_{w}+\frac{\pi}{2}\right)}+\mathrm{A}_{n}^{\delta} H_{n}\left(k r_{\delta}\right)\right. \\
\times e^{i n \theta_{\delta 0}}\left(\sum_{\mu=1}^{\delta-1} \ldots \sum_{\mu=\delta+1}^{\Delta}, \ldots\right] \\
\left.\times \sum_{m=-\infty}^{\infty} A_{n}^{\mu} H_{n+m}\left(k r_{\delta}\right) J_{m}\left(k r_{\delta}\right) e^{i m\left(\theta_{\delta \mu}-\theta_{\delta}\right)} e^{i n \theta_{\mu \delta}}\right\} e^{-i \omega t}
\end{gathered}
$$




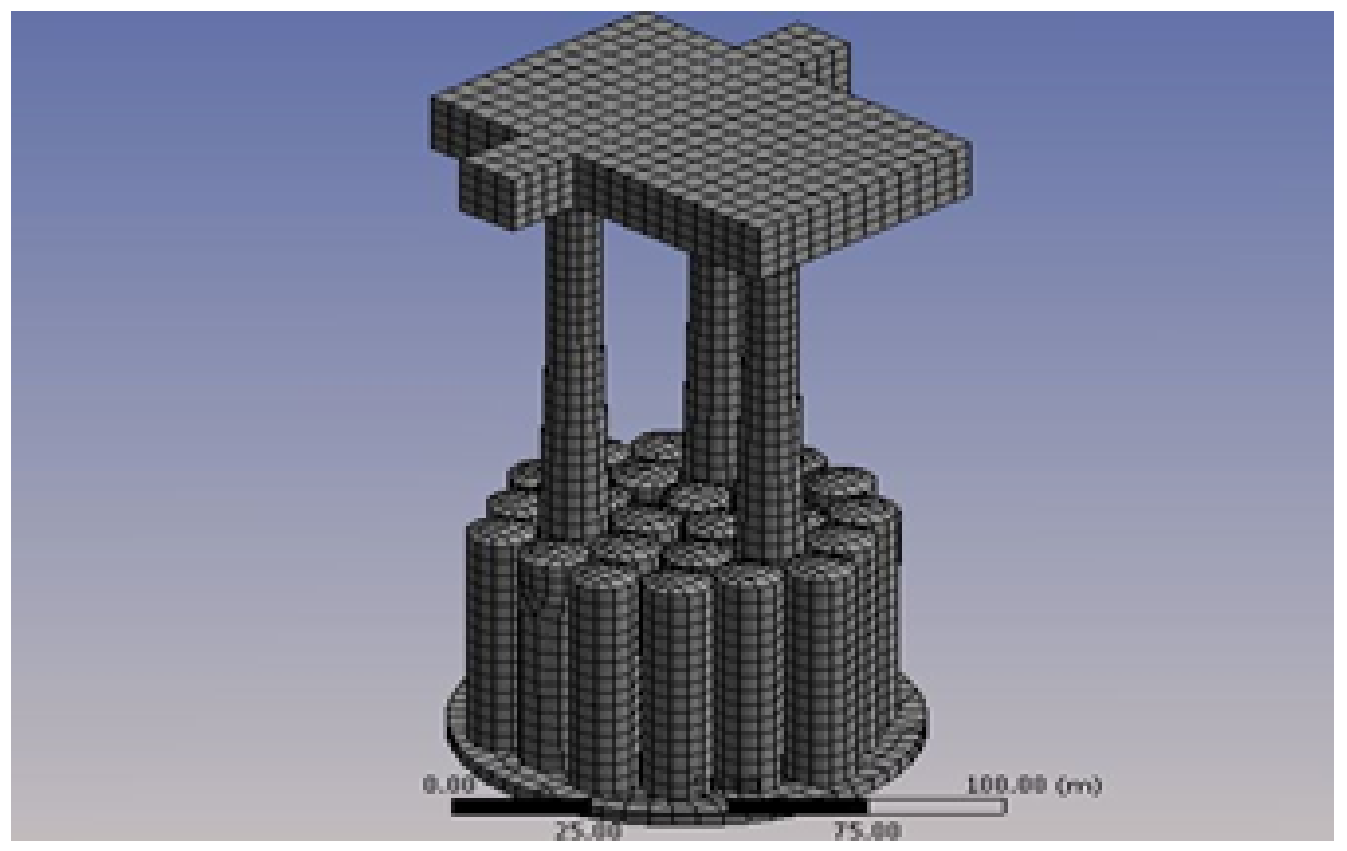

Figure 3. Modeling in AQWA Gravity Base Structure GBS for Three hollow columns platform

\section{RESULTS AND DISCUSSION}

3.1. The analysis of the hydrodynamic force for one column and Three hollow columns platform

According to the analysis result of the hydrodynamic force for one column platform as Figure 4 to 6 , and Three hollow columns as Figure 7 to 9:

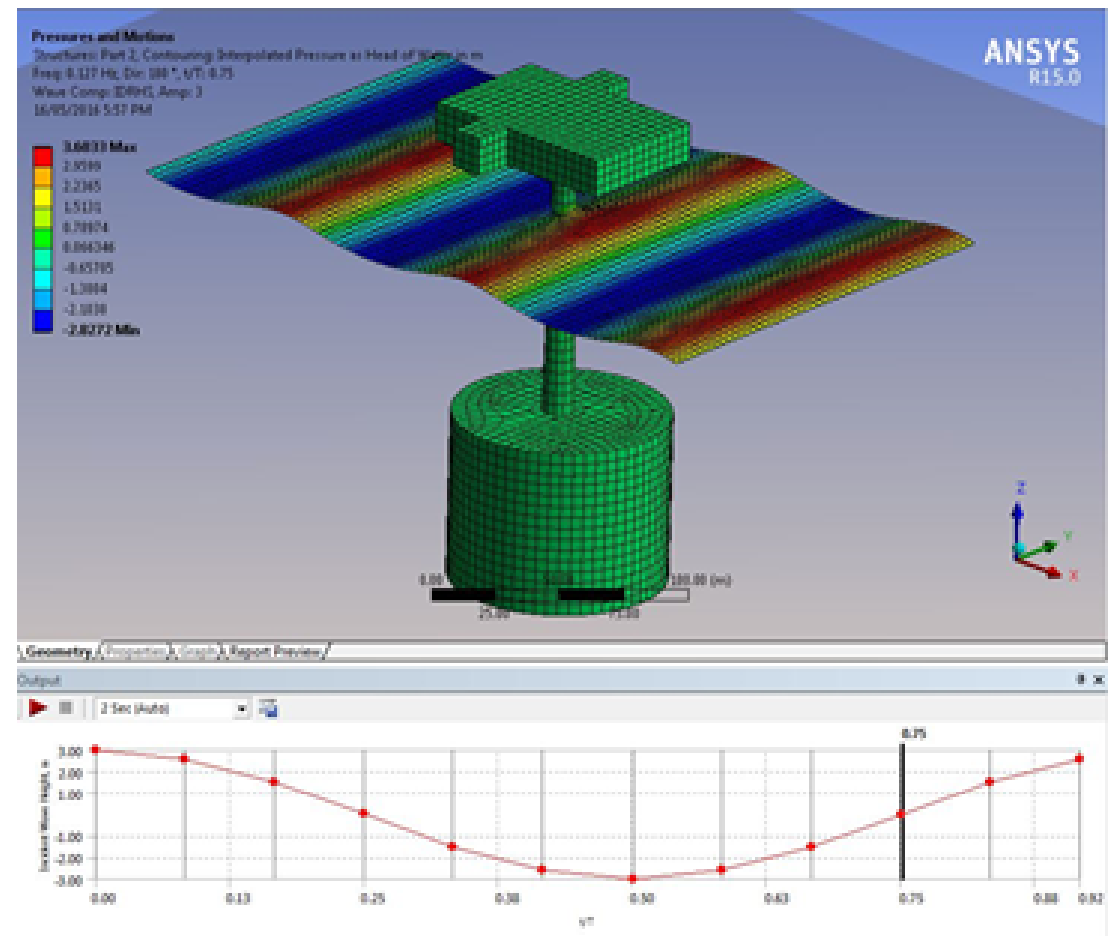

Figure 4. A view of the pressure caused by hydrodynamic forces in the Period 


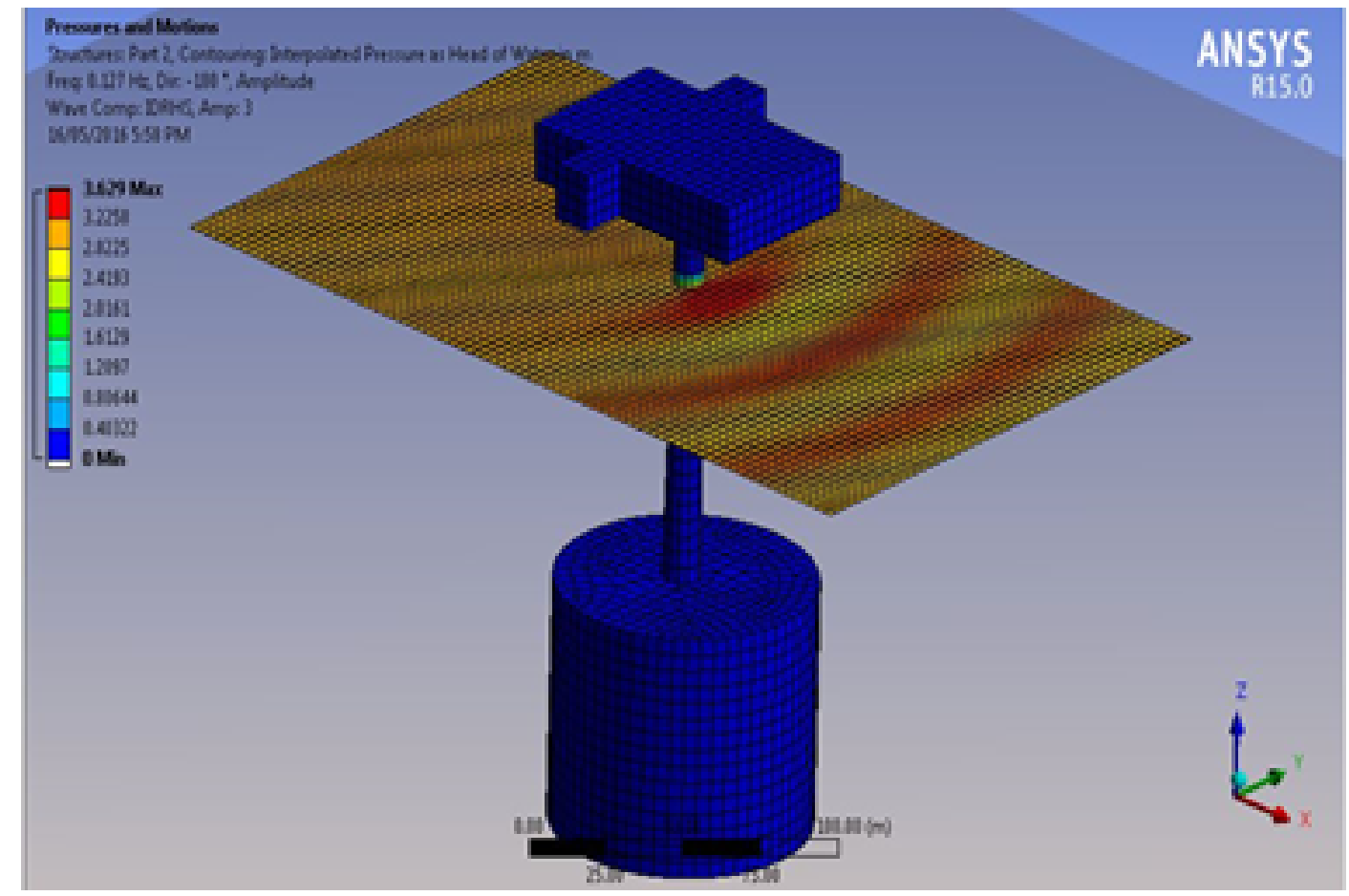

Figure 5. A view of the pressure caused by hydrodynamic forces in the frequency domain

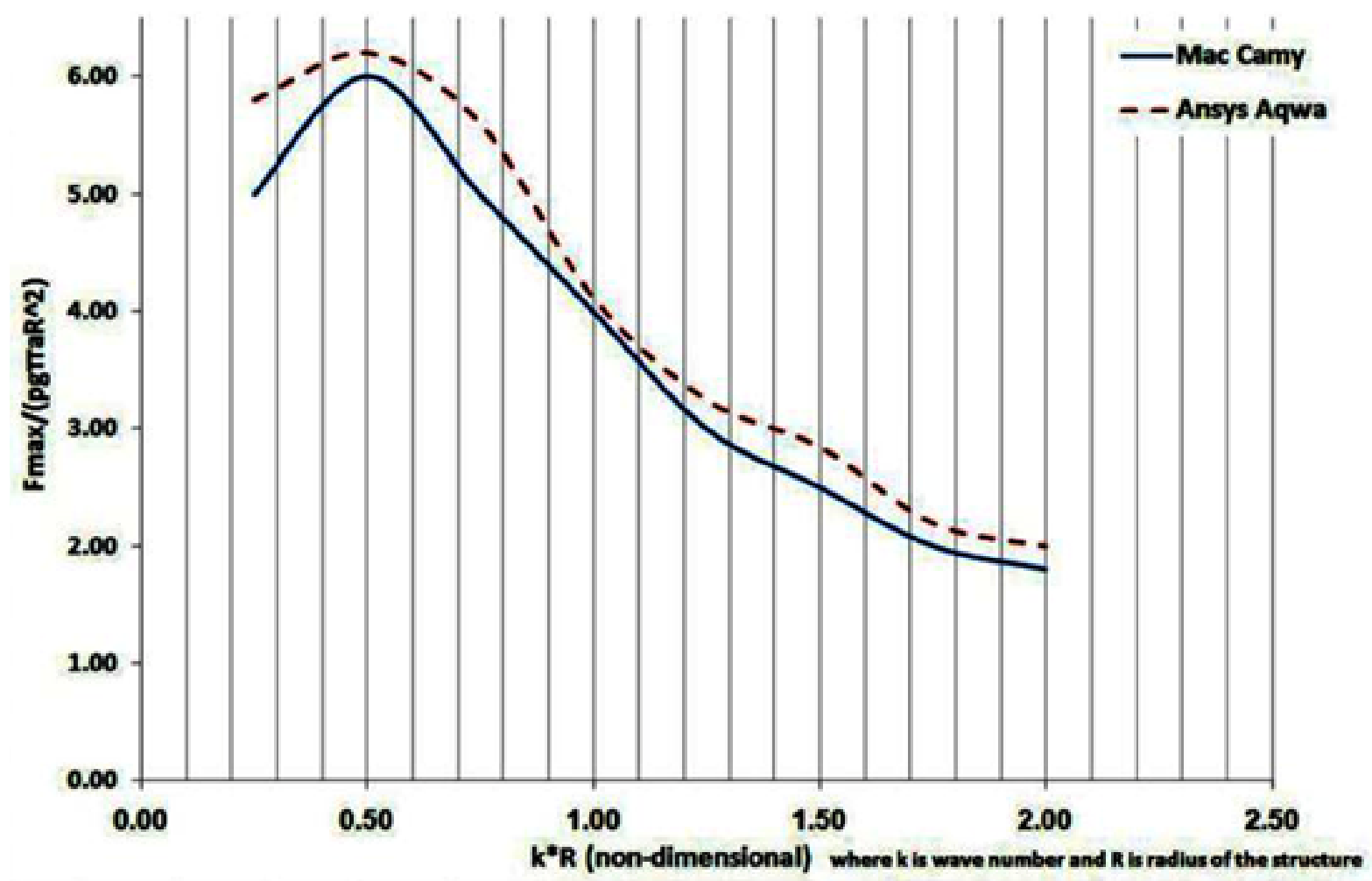

Figure 6. the Graph of maximum hydrodynamic force of wave based on k.D parameters for one column platform 


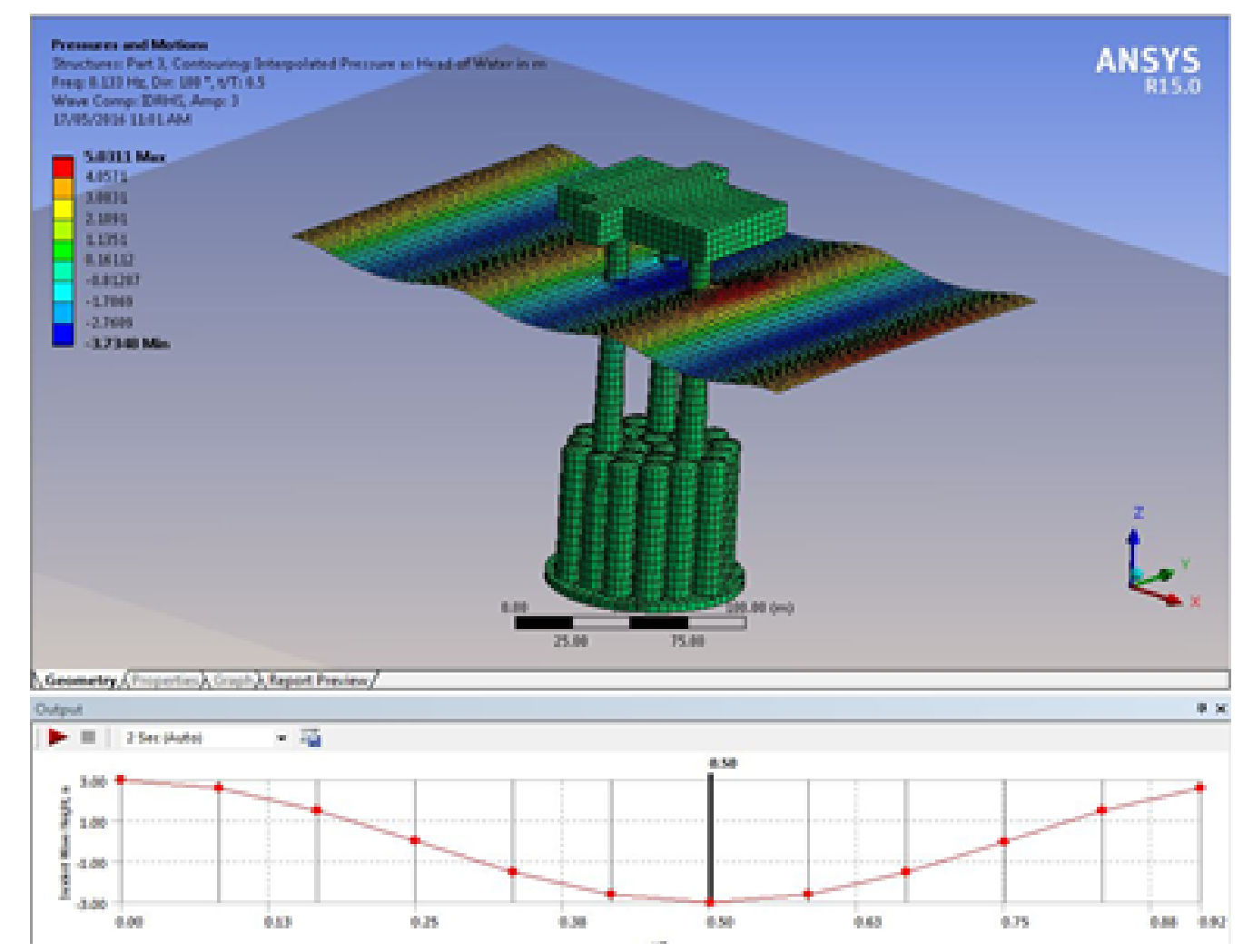

Figure 7. A view of the pressure caused by hydrodynamic forces in the Period

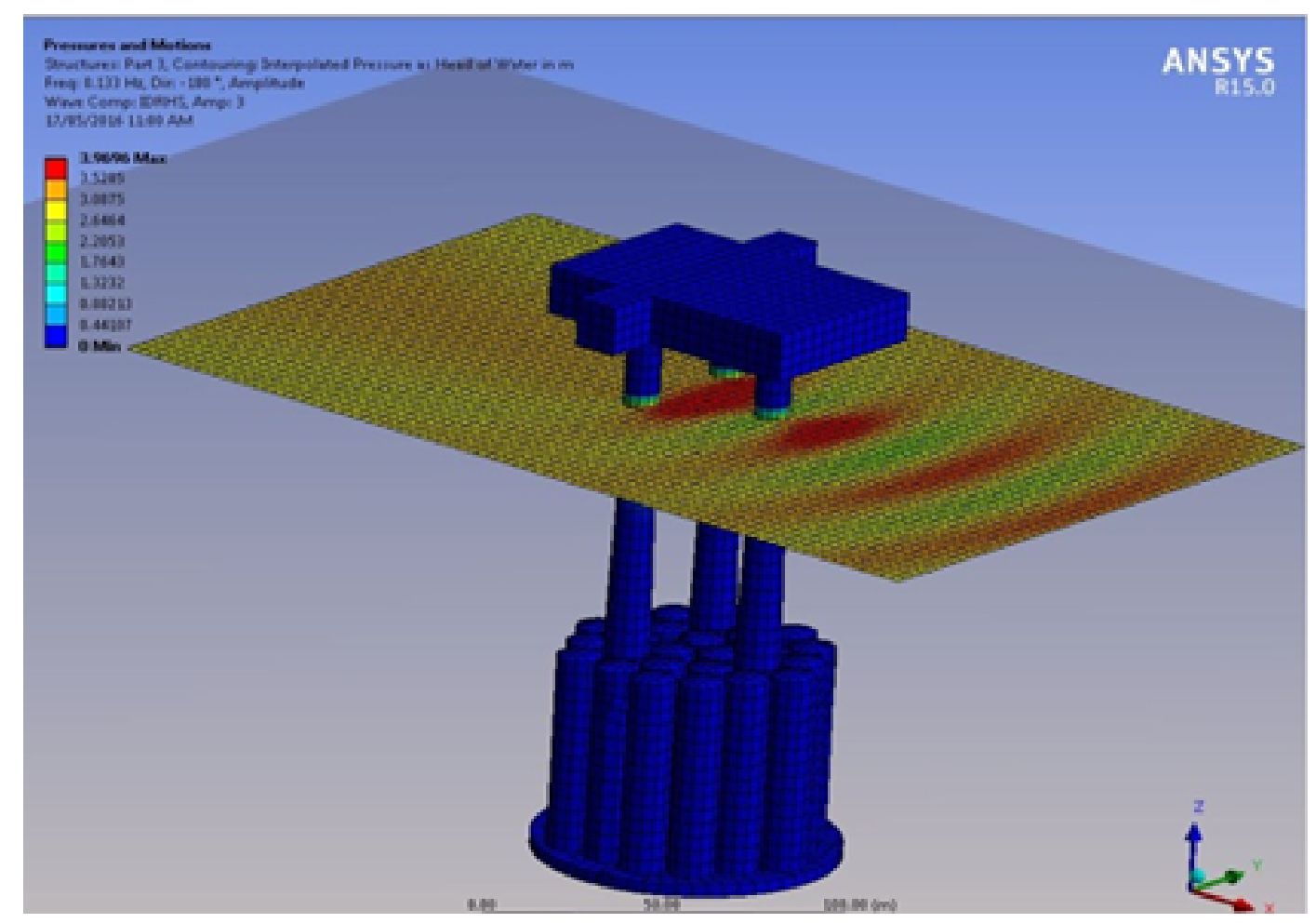

Figure 8. A view of the pressure caused by hydrodynamic forces in the frequency domain 


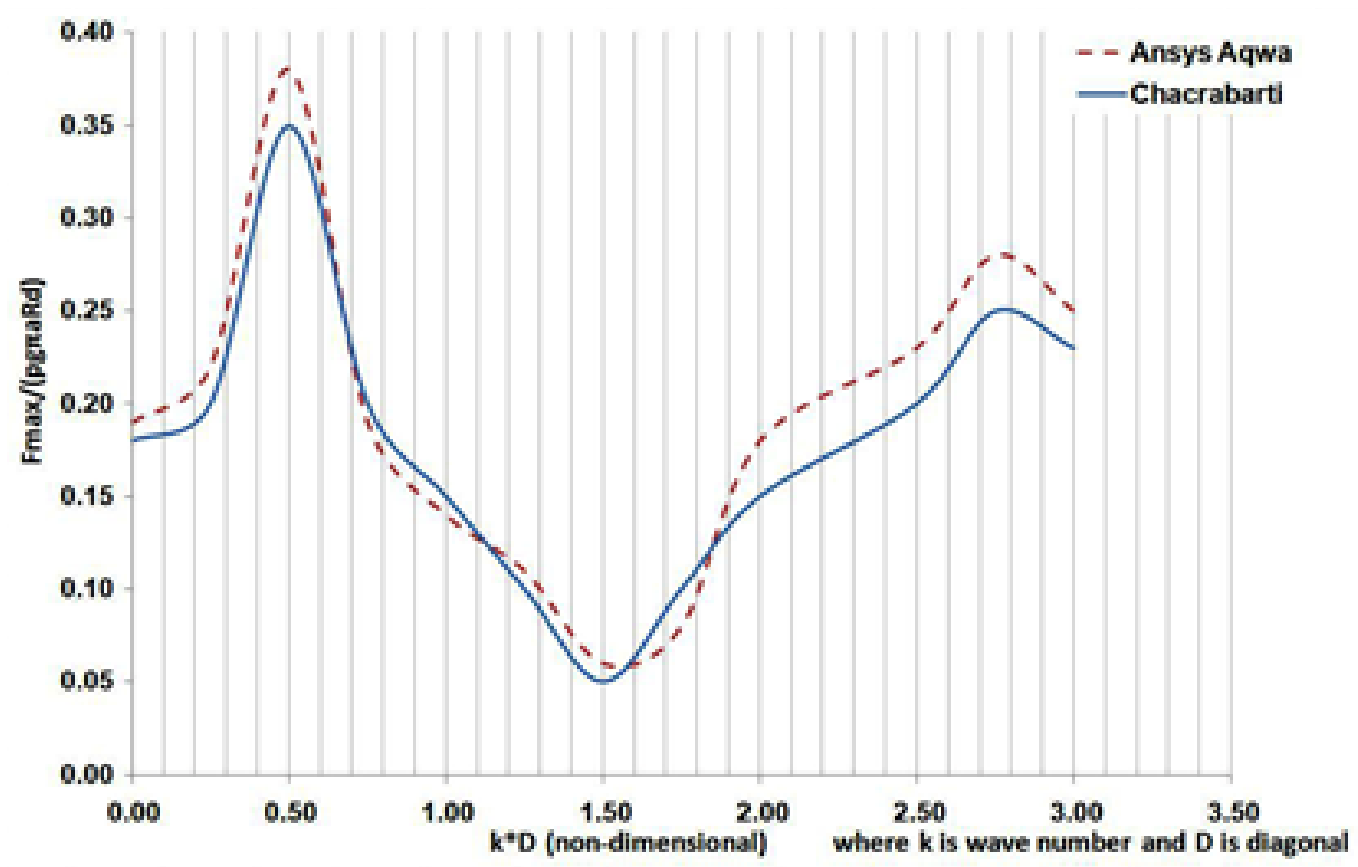

Figure 9. the Graph of maximum hydrodynamic force of wave based on k.D parameters for Three hollow columns platform

According to the maximum amount of nondimensional hydrodynamics force in the Three hollow columns platform, the maximum and the minimum amounts of free water level happen in the moments of $\mathrm{t}=\mathrm{T} / 2$ and $\mathrm{t}=0$ and in the one column platform, the maximum and the minimum amounts of free water level happen in the moments of $\mathrm{t}=\mathrm{T} / 4$. Also, the maximum and the minimum values, in the force curve are attributed to waves whose half the wavelength is a multiplication of the distance between the bases of the platform.

\subsection{Changes of moment and response of structures to the height}

To obtain the moment of wave, we have to accumulate the force in all parts of the platform (platform's base that wave power is applied to it).

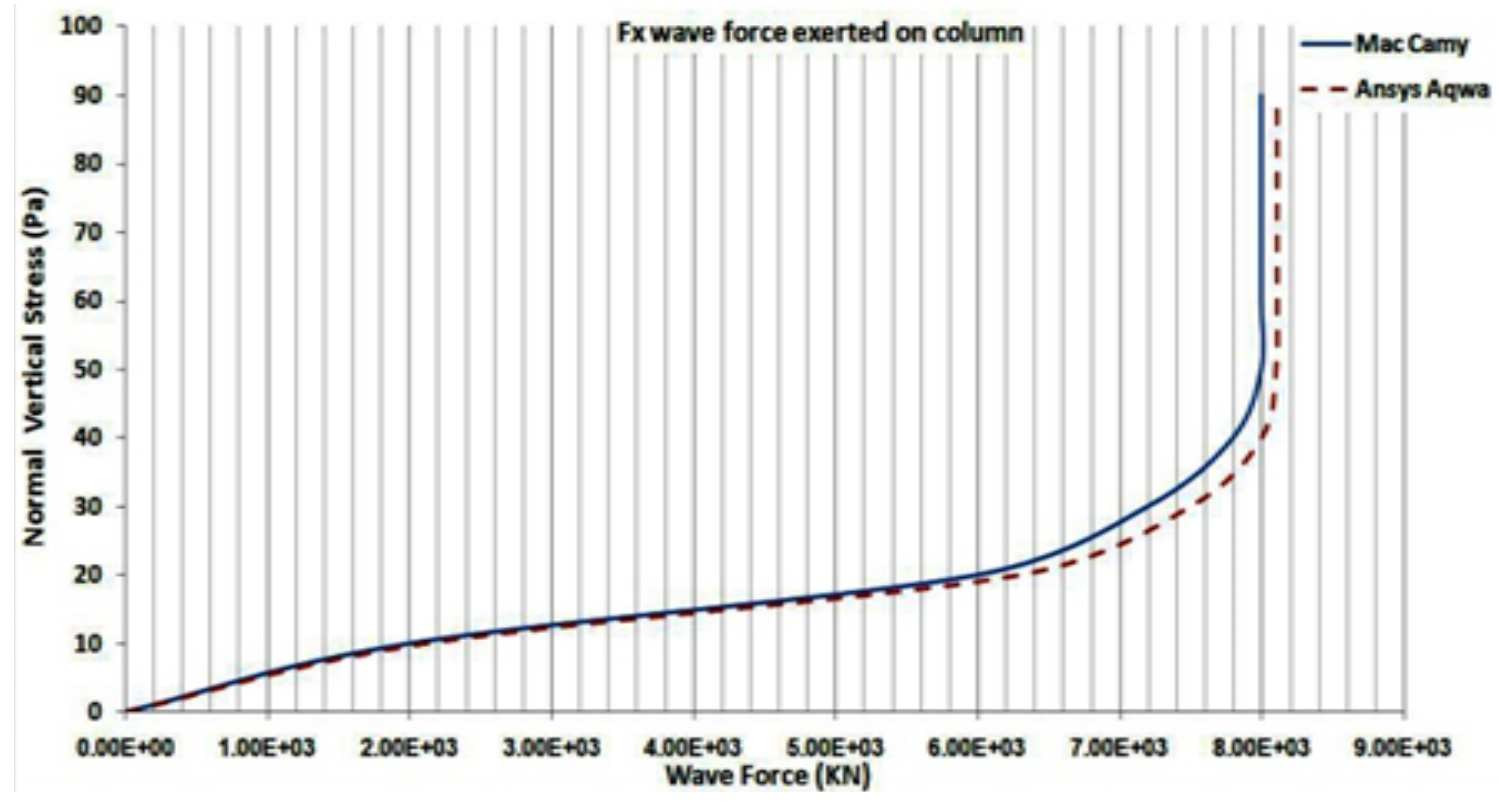

Figure 10. The force changes of the incoming waves on the base of gravity platform from the water surface to any specified depth 


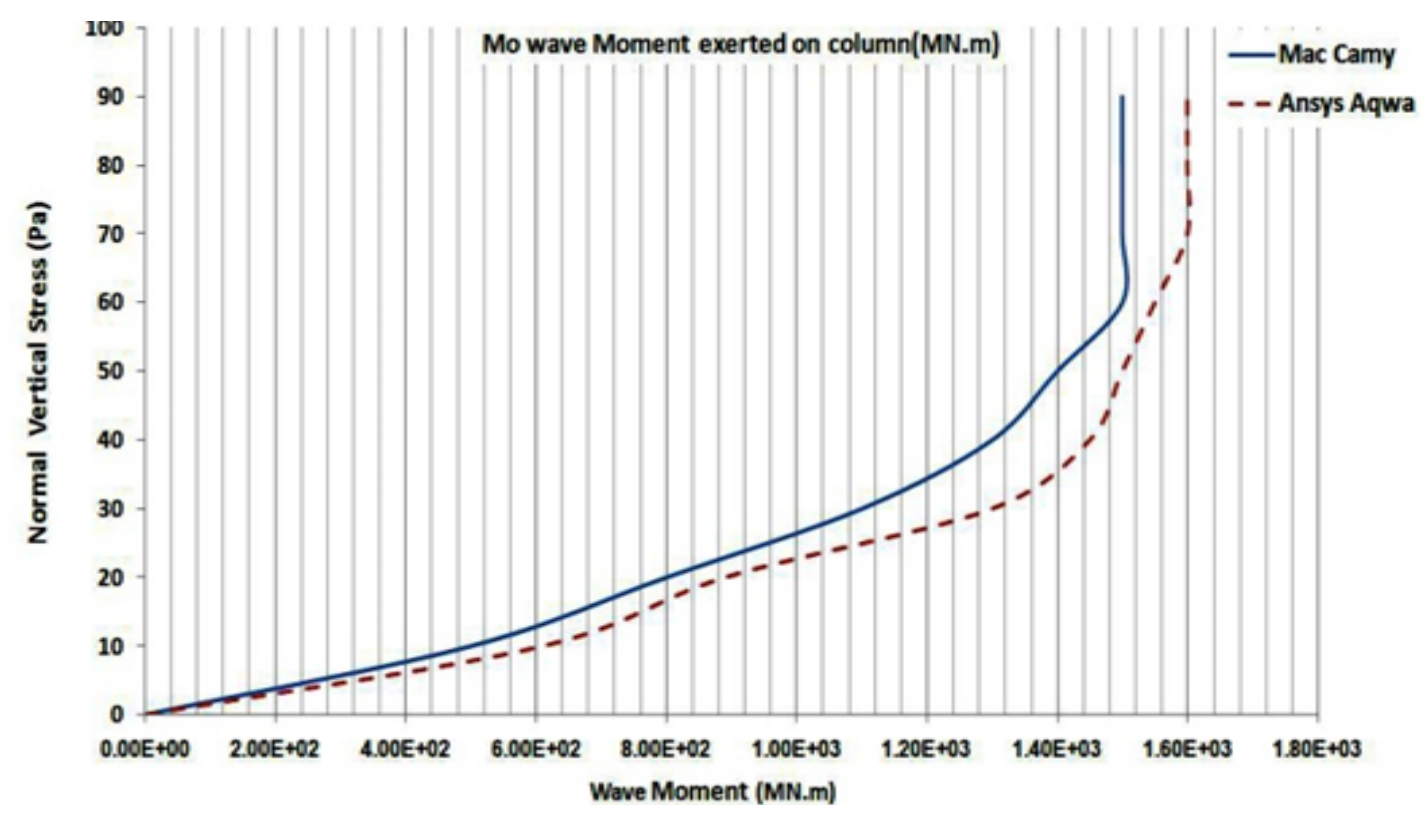

Figure 11. Changes of wave moment on the bases of the gravity platform from the water surface to any specified depth of platform

The results according Figure 10 and 11, showed that with the increasing of the depth, the impact of wave's force and moment on the base of platform are reduced through exponential relationship. The amount of force and total moment are inclined to a fixed value. The reductions are due to the effective depth that is equal to half the wavelength; so that it reduces the amount of force and moment to a small amount.

\subsection{Analysis of the effect of hydrodynamic wave parameters and structural response}

Analysis of the effect of hydrodynamic wave parameters and structural response of waves is of irregular and random nature, and analysis of related time histories for assessment of offshore structures is extremely important. The time history of waves demonstrates two substantial characteristics: the major wave height and frequency content. In this approach, numerical methods for analyzing structural response to irregular waves using JONSWAP spectrum are examined, and effect of different wave parameters is studied on hydrodynamic forces and response of a three-abutment gravity platform according Figure 12 to 14. 


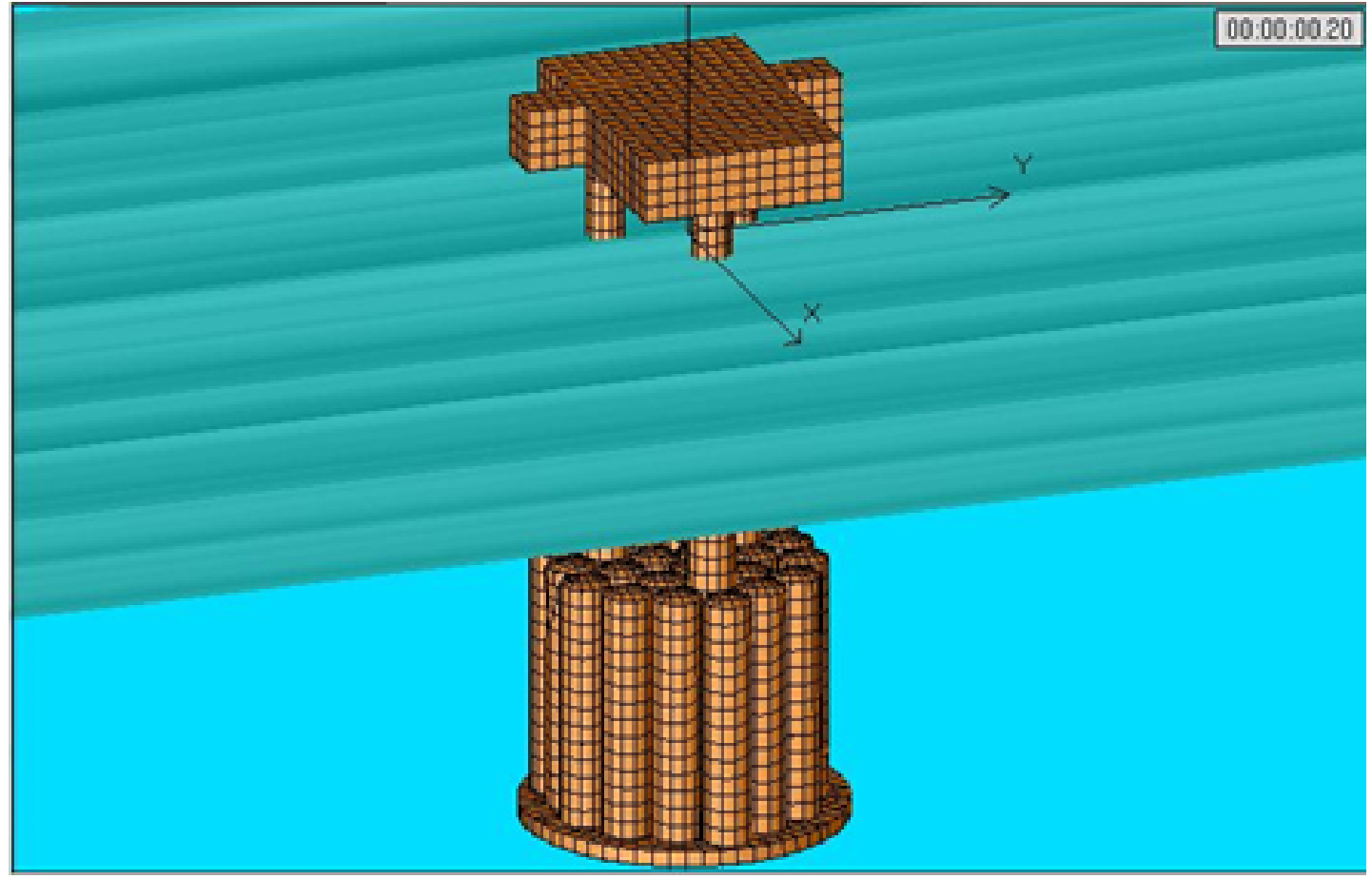

Figure 12. A view of interference of an irregular wave with a gravity platform over time

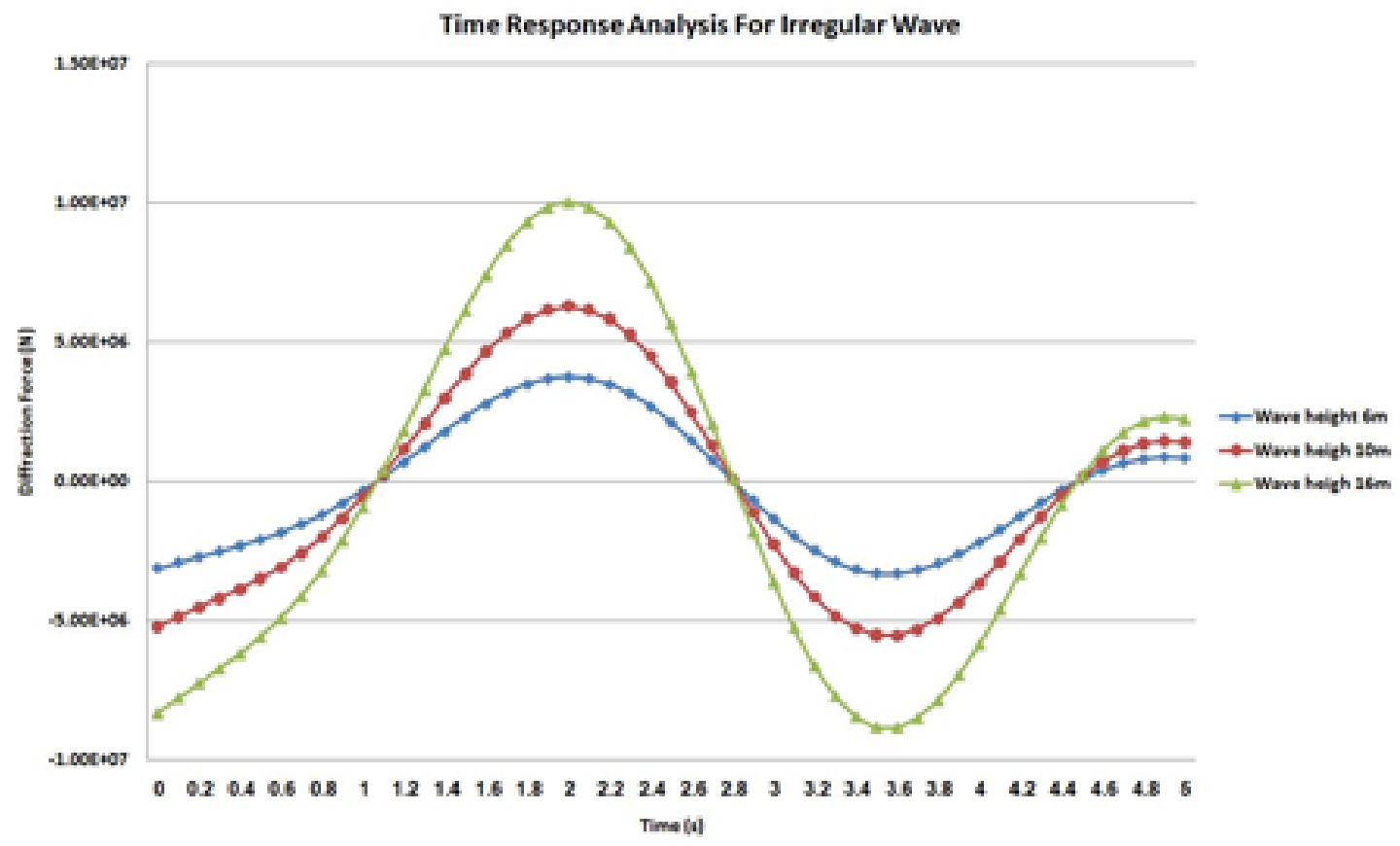

Figure 13. Changes in the diffraction force curve of the irregular wave in the $\mathrm{X}$ direction over time with different wave heights 


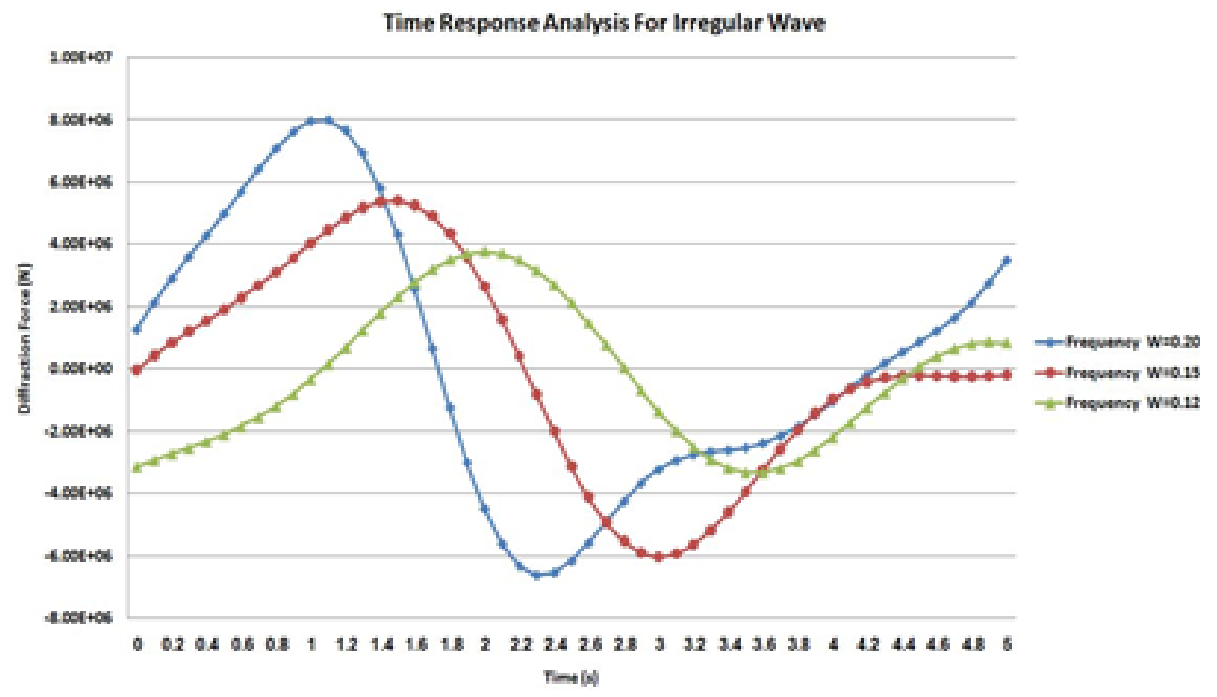

Figure 14. Changes in the diffraction force curve of the irregular wave in the $\mathrm{X}$ direction over time with different wave lengths

Based on the curves fitted to the data of the fluctuation angle, sustainability of the platform in the rocking motion can be thoroughly and completely investigated.

\subsection{The analysis of platform stability index}

Finally, in order to analyze the stability of the platform across the range of wave changes, the stability index of the platform was defined as the ratio of the overturning moment to restoring moment. Look at the following equation:
$S I=\frac{\mathrm{M}_{\mathrm{o}}}{\mathrm{kx} \theta}$

Mo is the turnover moment and $\mathrm{kx} \theta$ is the restoring moment obtained through the multiplication of $\mathrm{K}$ stiffness of soil and $\theta$ as the fluctuation range of the structure.

Based on the figure (15) it is realized that the platform in a rocking fluctuation is within the safe limits. According to the analysis result of platform stability for Three hollow columns platform:

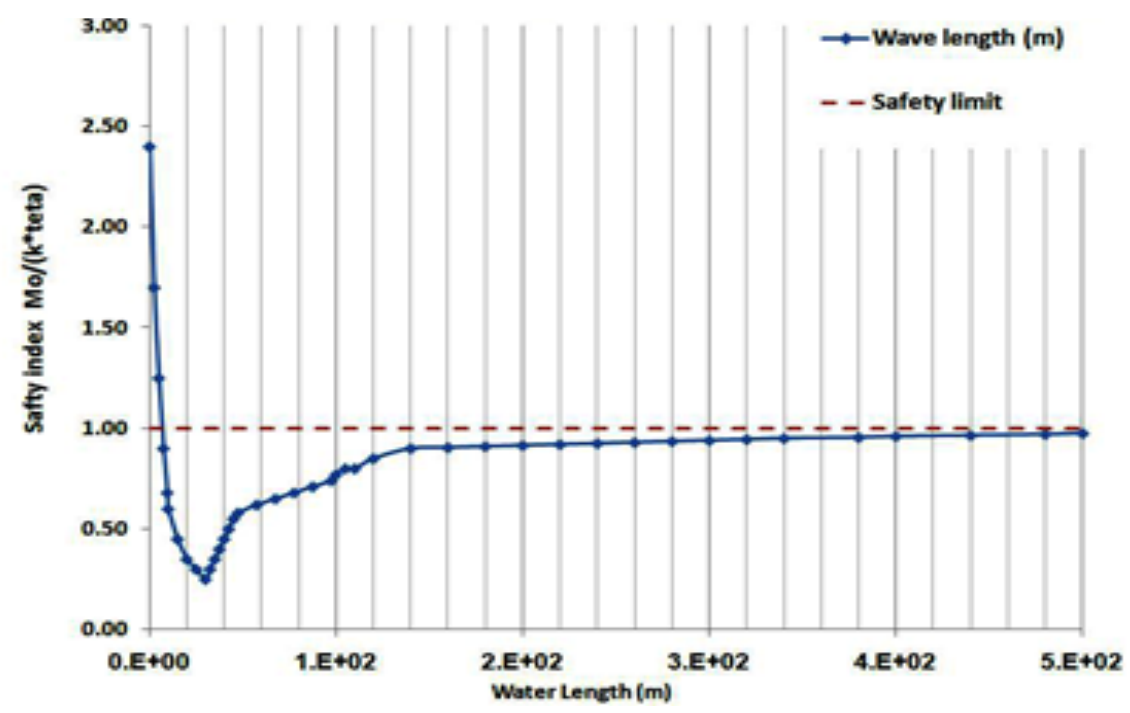

Figure 15. Structural changes of the frequency of the fluctuation angle 
Finally, in order to analyze the stability of the platform across the range of wave changes, the stability index of the platform was defined as the ratio of the overturning moment to restoring moment.

\section{CONCLUSION}

The results showed that with the increasing of the depth, the impact of wave's force and moment on the base of platform are reduced through exponential relationship. The amount of force and total moment are inclined to a fixed value. The reductions are due to the effective depth that is equal to half the wavelength; so that it reduces the amount of force and moment to a small amount.

Based on the maximum amount of non-dimensional hydrodynamics force in the Three hollow columns platform, the maximum and the minimum amounts of free water level happen in the moments of $t=$ $\mathrm{T} / 2$ and $\mathrm{t}=0$ and in the one column platform, the maximum and the minimum amounts of free water level happen in the moments of $\mathrm{t}=\mathrm{T} / 4$. Also, the maximum and the minimum values, in the force curve are attributed to waves whose half the wavelength is a multiplication of the distance between the bases of the platform. The results also suggest that the response of the rocking motion of gravity platform shows significant changes in relation to height and wavelength. Based on the curves fitted to the data of the fluctuation angle, sustainability of the platform in the rocking motion can be thoroughly and completely investigated. Regarding the regular waves, different analytical methods in the different structural conditions had good agreement with numerical data. So we can use the numerical methods for analyzing the structural response to irregular waves in dealing with gravity platform.

\section{REFERENCES}

1. Dean, E. T. R., "Offshore Geotechnical Engineering", 1st edition, Thomas Telford Limited, 2010.

2. Wilson, J. F., "Dynamics of Offshore Structures", John Wiley \& Sons, 2003.

3. McCormick, M. E., "Ocean Engineering Mechanics”, Cambridge University Press, 2010.

4. Mogridge, G. R., Jamieson, W. W., "Wave Forces on Large Circular Cylinders:A Design Method", Hydraulics Laboratory, National Research Council of Canada, Report MH-111, 1976.

5. Stansberg, C. T., Baarholm, R., Fokk, T., Gudmestad, O. T., Haver, S., "Wave amplification and possible deck impact on gravitybased structure in probability extreme crest heights", 23rd international conference on offshore mechanics and arctic engineering, 2004.

https://doi.org/10.1115/OMAE2004-51506

6. Chacrabarti S.K., "Hydrodynamics Of Offshore Structures ",(1994),Mechanics Publication.

7. Barati M.," Numerical model diffraction waves around the base of calculation force of the waves offshore platforms ",(2002), Thesis of Tehran Univer 